\title{
Modelling of attitude error in vector magnetic data: application to Ørsted data
}

\author{
R. Holme \\ GeoForschungsZentrum Potsdam, Telegrafenberg, D-14473 Potsdam, Germany
}

(Received March 3, 2000; Revised July 14, 2000; Accepted July 17, 2000)

\begin{abstract}
Analysis of data from the Ørsted satellite indicates that the largest source of error arises from an anisotropic attitude uncertainty, related to poorly determined rotation about the axis of the star imager. For two data sets from May and December 1999, I quantify this error, and review and apply a formalism designed to allow for this problem. I argue that, when modelled correctly, this attitude uncertainty should not significantly damage the main field models obtained from Ørsted data.
\end{abstract}

\section{Introduction}

In modelling of the geomagnetic field, it is customary to treat the errors on the data as uncorrelated, although this is generally far from the case. The full treatment of error correlations requires the inversion of a data by data matrix, which is impractical given the quantity of data required for high quality field modelling (although see Rygaard-Hjalsted et al. (1997) for the application of sparse matrix techniques towards this goal). However, one form of error correlation can be treated easily: when different components of the field measured at the same place and time are correlated. Such local correlation can be allowed for easily at no additional numerical cost, and it has been shown that so doing can lead to significant improvements in field modelling. Two cases have been considered in detail thus far: anisotropy in the crustal field error for main field modelling (Holme and Jackson, 1997), and the effect of attitude uncertainty in spacecraft magnetometry (Holme and Bloxham, 1995, 1996).

The consideration of these methods has received new urgency from study of data from the Danish magnetic satellite Ørsted. This satellite was launched on February 23rd, 1999, and has returned much high quality data. More details of the mission and the field models which have been produced from the data can be found elsewhere in this issue. However, one aspect of the data still requires detailed attention. Ørsted carries only one star camera, which provides highly accurate pointing information but lower resolution in its rotational direction. Preflight estimates of the uncertainties suggested that this effect would not be important (see, for example, Holme and Bloxham, 1996), but here, for two data sets from May and December 1999, I estimate the rotational error to be of order $60^{\prime \prime}$, by far the largest contributor to the vector data error budget. It is clear that to avoid degrading the field models and subsequent science derived from Ørsted data, this effect must be accounted for properly.

As such, it is timely to reconsider the modelling of attitude error. I first summarise the basic field modelling framework,

Copy right(c) The Society of Geomagnetism and Earth, Planetary and Space Sciences (SGEPSS); The Seismological Society of Japan; The Volcanological Society of Japan; The Geodetic Society of Japan; The Japanese Society for Planetary Sciences. and the formalism for modelling the attitude error. This material has appeared previously; however, it is hoped that a more developed understanding of the problem will make this new presentation useful. I follow this with results from simulations that quantify the degradation of field models from attitude error. The method is then applied to the two Ørsted datasets, and the magnitude of the attitude error estimated. To aid other workers in assessing the usefulness and importance of the method, I use simulations to estimate the model contamination caused by ignoring the error correlations. I conclude that correct modelling of attitude error in Ørsted data is important, and provide a recommendation for future mission management to minimise its effects.

\section{Determining a Field Model}

In a current-free region, the magnetic field can be expressed as $\mathbf{B}=-\nabla \Phi$, where the scalar potential $\Phi$ satisfies $\nabla^{2} \Phi=0$. In spherical coordinates, the potential can be represented by a spherical harmonic expansion of the form

$$
\begin{aligned}
\Phi= & a \sum_{l=1}^{\infty}\left(\frac{a}{r}\right)^{l+1} \sum_{m=0}^{l} P_{l}^{m}(\cos \theta) \\
& \cdot\left[g_{l}^{m} \cos (m \phi)+h_{l}^{m} \sin (m \phi)\right] \\
& +a \sum_{l=1}^{\infty}\left(\frac{r}{a}\right)^{l} \sum_{m=0}^{l} P_{l}^{m}(\cos \theta) \\
& \cdot\left[q_{l}^{m} \cos (m \phi)+s_{l}^{m} \sin (m \phi)\right]
\end{aligned}
$$

where $a$ is the radius of the Earth, and $(r, \theta, \phi)$ is a geocentric spherical coordinate system. $P_{l}^{m}(\cos \theta)$ are Legendre polynomials, by convention Schmidt normalised, so that

$$
\int_{\phi=0}^{2 \pi} \int_{\theta=0}^{\pi}\left(P_{l}^{m}(\cos \theta) \cos m \phi\right)^{2} \sin \theta d \theta d \phi=\frac{4 \pi}{2 l+1} .
$$

Measurements of the magnetic field are used to estimate the so-called Gauss coefficients $\left(\left\{g_{l}^{m}, h_{l}^{m}\right\}\right.$ for internal sources, and $\left\{q_{l}^{m}, s_{l}^{m}\right\}$ for external sources), which then uniquely define the geomagnetic field outside the source regions. After truncating the series in Eq. (1), the Gauss coefficients are ob- 
tained by a least-squares fit to the available data, minimising

$$
\mathbf{e}^{\mathrm{T}} \mathbf{C} \mathbf{e}^{-1} \mathbf{e}
$$

where $\mathbf{C}_{\mathbf{e}}$ is the data error covariance matrix, with elements

$$
\left(C_{e}\right)_{i j}=\operatorname{cov}\left(e_{i}, e_{j}\right)
$$

and the error vector $\mathbf{e}$ is given by

$$
\mathbf{e}=\boldsymbol{\gamma}-\mathbf{A m}
$$

where $\mathbf{A}$ is an operator calculated from Eq. (1) relating the data vector $\gamma$ to the model vector $\mathbf{m}$. For the linear problem (see, e.g., Gubbins and Bloxham, 1985, for the extension to nonlinear modelling) the maximum likelihood solution $\hat{\mathbf{m}}$ is obtained by solving the equation

$$
\left(\mathbf{A}^{\mathrm{T}} \mathbf{C}^{-1} \mathbf{A}\right) \hat{\mathbf{m}}=\mathbf{A}^{\mathrm{T}} \mathbf{C} \mathbf{e}^{-1} \boldsymbol{\gamma} .
$$

The field modelling process is inherently non-unique; there are an infinite number of parameters $\left\{g_{l}^{m}, h_{l}^{m}, q_{l}^{m}, s_{l}^{m}\right\}$ to fit an unavoidably finite number of data. This problem is best dealt with by regularisation or damping (Whaler and Gubbins, 1981; Shure et al., 1982; Gubbins and Bloxham, 1985), but here I adopt the simpler approach (reasonable when the data distribution is fairly even) of truncating the spherical harmonic series. I truncate at degree $\operatorname{lmax}=13$ for internal sources, and degree 1 for external sources. Field models from Magsat data (for example, that of Cain et al., 1989) suggest that at Ørsted altitude the rms magnitude of higher degree internal field components (of crustal origin) will be of order only $2 \mathrm{nT}$, while previous models have suggested that for near-Earth data, higher-degree external terms are not significant (e.g., Langel and Estes, 1985).

\section{Theory}

Modelling of attitude error requires construction of the correct covariance matrix $\mathbf{C}_{e}$. Neglecting correlations between measurement points, consider the covariance of a particular orthogonal data triple. Uncertainty in the orientation of the measured reference frame (variance $\psi^{2}$ ) introduces errors in vector components of the magnetic field $\mathbf{B}$, but not its magnitude. To first order, the error is purely perpendicular to the field, as sketched in Fig. 1. If $\sigma^{2}$ is the instrument variance for each orthogonal component, and I the $3 \times 3$ identity, the data error covariance matrix for a vector triple of magnetic field components is, as given by Holme and Bloxham (1995) (henceforth Paper 1)

$$
\mathbf{C}=\sigma^{2} \mathbf{I}+\psi^{2}\left(B^{2} \mathbf{I}-\mathbf{B B}^{\mathrm{T}}\right)
$$

(Valid for $\sin \psi \approx \psi, \sigma \gg B \psi^{2}$ (Holme and Bloxham, 1996, henceforth, Paper 2)). This matrix is most easily applied by writing it in terms of vector outer products or dyadics:

$$
\begin{aligned}
\mathbf{C}= & \sigma^{2} \frac{\mathbf{B B}^{\mathrm{T}}}{B^{2}}+\left(\sigma^{2}+B^{2} \psi^{2}\right) \frac{(\hat{\mathbf{n}} \wedge \mathbf{B})(\hat{\mathbf{n}} \wedge \mathbf{B})^{\mathrm{T}}}{(\hat{\mathbf{n}} \wedge \mathbf{B})^{2}} \\
& +\left(\sigma^{2}+B^{2} \psi^{2}\right) \frac{(\mathbf{B} \wedge(\hat{\mathbf{n}} \wedge \mathbf{B}))(\mathbf{B} \wedge(\hat{\mathbf{n}} \wedge \mathbf{B}))^{\mathrm{T}}}{(\mathbf{B} \wedge(\hat{\mathbf{n}} \wedge \mathbf{B}))^{2}}
\end{aligned}
$$

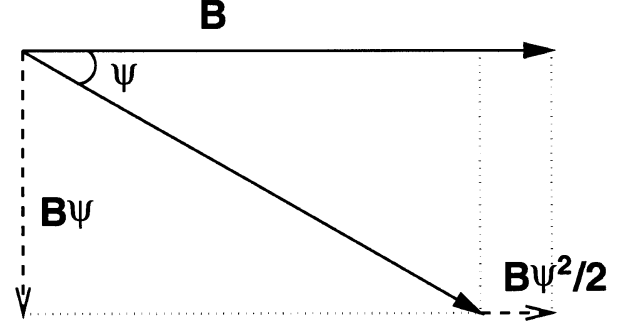

Fig. 1. The directional dependence of orientation error.

where $\hat{\mathbf{n}}$ is an arbitrary unit vector not parallel to $\mathbf{B}$. Although this representation may be unfamiliar, it is identical to the standard diagonalisation of a matrix by determination of its eigenvalues and eigenvectors, the latter being the unit vectors in the dyadics.

In Paper 2, this formalism was extended to cover the case of Ørsted, where the attitude uncertainty itself is anisotropic. It is assumed that this takes the form of a small angular uncertainty $\psi$ in rotation perpendicular to the camera bore sight (the pointing angle), and a larger rotational uncertainty $\chi$ about the bore sight. The covariance matrix is now

$$
\begin{aligned}
\mathbf{C}= & \sigma^{2} \frac{\mathbf{B B}^{\mathrm{T}}}{B^{2}} \\
& +\left(\sigma^{2}+(\hat{\mathbf{n}} \wedge \mathbf{B})^{2} \chi^{2}+(\hat{\mathbf{n}} \cdot \mathbf{B})^{2} \psi^{2}\right) \frac{(\hat{\mathbf{n}} \wedge \mathbf{B})(\hat{\mathbf{n}} \wedge \mathbf{B})^{\mathrm{T}}}{(\hat{\mathbf{n}} \wedge \mathbf{B})^{2}} \\
& +\left(\sigma^{2}+B^{2} \psi^{2}\right) \frac{(\mathbf{B} \wedge(\hat{\mathbf{n}} \wedge \mathbf{B}))(\mathbf{B} \wedge(\hat{\mathbf{n}} \wedge \mathbf{B}))^{\mathrm{T}}}{(\mathbf{B} \wedge(\hat{\mathbf{n}} \wedge \mathbf{B}))^{2}}
\end{aligned}
$$

where $\hat{\mathbf{n}}$ is no longer arbitrary, but is a unit vector in the direction of the star camera bore sight (assumed not parallel to B). Note that to calculate $\mathbf{C}$, only the star camera direction and the measured magnetic field are required - no additional details of the spacecraft trajectory or motion are necessary. If the star camera axis was aligned with the field, and the appropriate limits were taken to obtain the unit vectors, uncertainty about this axis would have no first order effect on the field measurement. If the field is perpendicular to the star camera (so that $(\hat{\mathbf{n}} \wedge \mathbf{B})^{2}=B^{2}$ and $\left.\hat{\mathbf{n}} . \mathbf{B}=0\right)$, then the error is maximised, although note that it acts only in one of the error eigendirections, so even in this case the expected error is less severe than if this large attitude error were isotropic. To emphasise this point, assume that only the rotational error is significant, so that $\psi=0$. In this case, the covariance matrix can be written in a particularly simple form

$$
\mathbf{C}=\sigma^{2} \mathbf{I}+\chi^{2}(\hat{\mathbf{n}} \wedge \mathbf{B})(\hat{\mathbf{n}} \wedge \mathbf{B})^{\mathrm{T}} .
$$

However, to obtain the matrix inverse, required in field modelling (Eq. (6)), it is easiest to return to the dyadic form. Because the dyadics are orthogonal and normalised to unit length, the inverse of the covariance matrix (9) is particularly simply expressed as

$$
\begin{aligned}
\mathbf{C}^{-1}= & \frac{1}{\sigma^{2}} \frac{\mathbf{B B}^{\mathrm{T}}}{B^{2}}+\frac{1}{\left(\sigma^{2}+(\hat{\mathbf{n}} \wedge \mathbf{B})^{2} \chi^{2}+(\hat{\mathbf{n}} . \mathbf{B})^{2} \psi^{2}\right)} \\
& \cdot \frac{(\hat{\mathbf{n}} \wedge \mathbf{B})(\hat{\mathbf{n}} \wedge \mathbf{B})^{\mathrm{T}}}{(\hat{\mathbf{n}} \wedge \mathbf{B})^{2}}
\end{aligned}
$$




$$
\begin{aligned}
& +\frac{1}{\left(\sigma^{2}+B^{2} \psi^{2}\right)} \\
& \cdot \frac{(\mathbf{B} \wedge(\hat{\mathbf{n}} \wedge \mathbf{B}))(\mathbf{B} \wedge(\hat{\mathbf{n}} \wedge \mathbf{B}))^{\mathrm{T}}}{(\mathbf{B} \wedge(\hat{\mathbf{n}} \wedge \mathbf{B}))^{2}} .
\end{aligned}
$$

This expression provides the inverse data covariance matrix for a triple of vector data. The full data covariance matrix (Eq. (4)) will be block diagonal, with $3 \times 3$ blocks as calculated from Eq. (11).

It is possible to extend this formalism further in several ways. While not relevelent for the current application, for completeness I discuss two such extensions in appendices; in Appendix A, the simultaneous consideration of vector and scalar data, and in Appendix B the use of directional information only from three component data.

\section{How Damaging is Orientation Uncertainty?}

Papers 1 and 2, and the specific data analysis later in this paper, concentrate on the expected improvement in the magnetic field model to be achieved by the correct treatment of attitude error. However, it is also important to quantify the significance of attitude error when correctly treated. I investigate this by means of numerical simulation. I construct a network of data points with approximately equal area distribution, and with altitude of $400 \pm 50 \mathrm{~km}$ (so as to mimic a Magsat-like satellite). On these points I calculate values of the vector field from a defined field model, and add independent Gaussian errors, both isotropic $\sigma$ and in attitude $\psi$. I then invert these simulated data for a magnetic field model. The quality of the model is defined by the deviation from the original model at the Earth's surface; as shown by Lowes (1966) amongst others a convenient measure of this is given by the integrated mean square field deviation at the Earth's surface

$$
\begin{aligned}
& \frac{1}{4 \pi a^{2}} \int\left(\mathbf{B}-\mathbf{B}^{0}\right)^{2} d \Omega \\
& \quad=\sum_{l=1}^{l m a x}(l+1) \sum_{m=0}^{l}\left(\left(g_{l}^{m}-g_{l}^{m 0}\right)^{2}+\left(h_{l}^{m}-h_{l}^{m 0}\right)^{2}\right)
\end{aligned}
$$

where the superscript 0 denotes the original model. The absolute value of this quantity is not especially meaningful; it will depend on the number of data points, the degree of truncation and other simulation details. However, the relative error between simulations is a useful measure of model quality, and turns out to be broadly independent of simulation details. (A clear demonstration of this can be seen in the related study of the influence of crustal field error in main field modelling in Holme and Jackson (1997) (subsequently Paper 3); for this case analytic expressions for the misfit have been derived by Dr. Frank Lowes.)

A set of such simulations is performed for a range of values of $\sigma$ and $\psi$, assuming the simplest case of isotropic attitude error, modelled by data covariance matrix (8). The results are averaged over 100 simulations, and the rms vector misfit plotted in Fig. 2. This figure suggests an approximate empirical linear relationship:

$$
\text { Misfit }=A(\sigma+0.2 B \psi)
$$

where $A$ is a constant depending on simulation details (number of data, harmonic truncation, etc.). $B \psi$ is generally taken

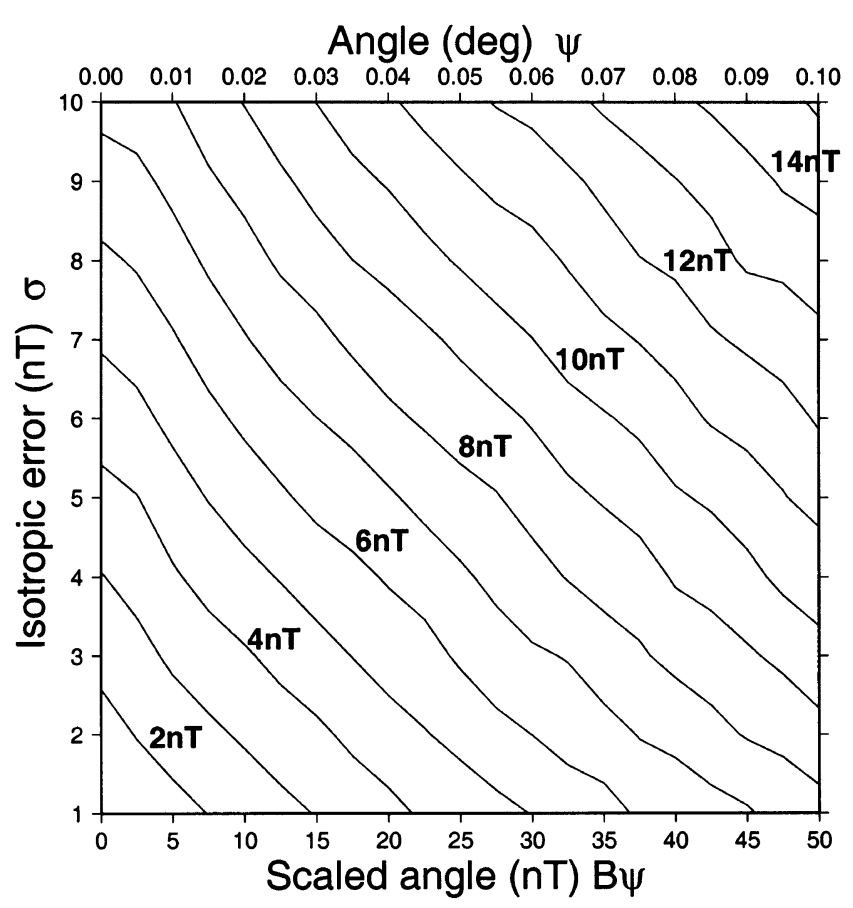

Fig. 2. Contour plot (in nT) of rms deviation of the vector field from the input model, averaged over the Earth's surface (Eq. (12)), for a range of applied data errors. Averages of 100 simulations with 2070 data triples grouped around $400 \mathrm{~km}$ (satellite altitude), least squares fit, truncation at degree 14. Scaled angle error calculated assuming a conservative value of satellite altitude $B=29000 \mathrm{nT}$. Exact misfit values depend on simulation details and so are not meaningful; relative values are robust.

as the estimate of the magnitude of field error; however, with this estimate these simulations show that correctly treated attitude error is only $20 \%$ as damaging as isotropic instrument error of the same magnitude. Further, for Ørsted, the damage from poorly determined attitude will be even less, as only one of the eigenvectors of the covariance matrix, rather than two as here, is badly affected (see Eq. (9)). However, as shown in Papers 1 and 2, should the error be modelled incorrectly as isotropic, much larger errors will result (see in particular figure 3, Paper 1).

\section{Data}

To seek evidence of attitude error in Ørsted data, I consider vector magnetometer (CSC) data from six days in May (10th-11th, 16th-17th, 21st-22nd), and from an extremely magnetically quiet period in December (20th-22nd). During these periods, satellite altitude varied between $690 \mathrm{~km}$ and $880 \mathrm{~km}$. The data (designated MAG-R) were hand corrected for timing errors by Nils Olsen and the Ørsted data centre. They are boxcar-averaged over $1.13 \mathrm{~s}$ intervals (the internal averaging period of the star camera). Nightside data only are selected to limit the influence of external fields. The data are heavily decimated in time, but weighted so as to give a higher sampling rate near the equator, and lower near the poles. About 20 data points from May 10 th have $K p=2 \mathrm{o}$; all other data are $K p=1+$ or less. No additional scalar data from the Overhauser magnetometer (OVM) are used; as a result the data sets are gappy, with a particularly large hole over the South Atlantic anomaly where the attitude information 

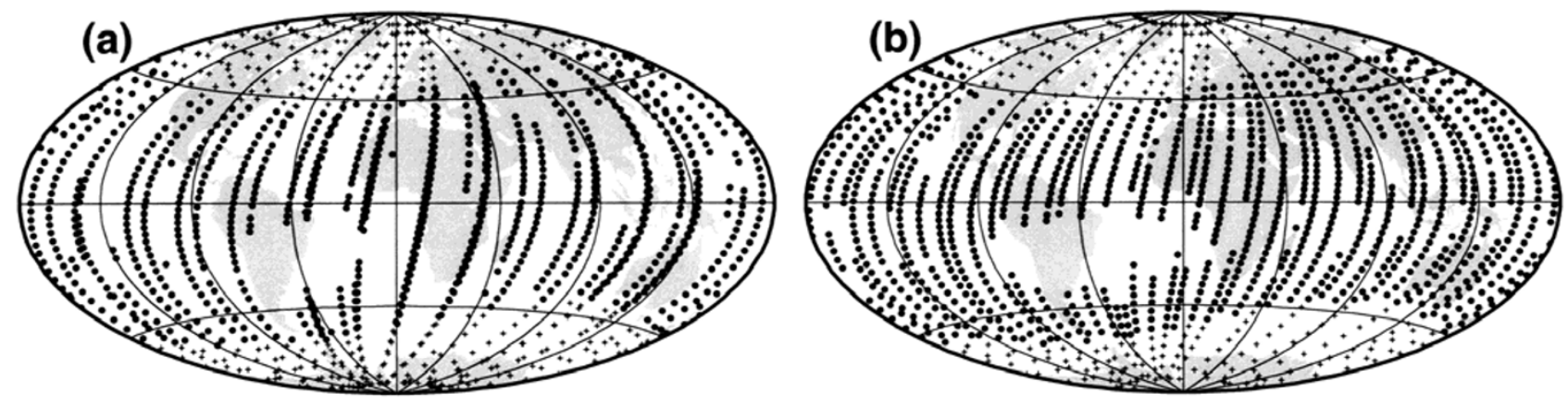

Fig. 3. Data distribution. (a) May-946 vector data triples (circles), 392 total-intensity data (crosses). (b) December-1169 vector data triples, 547 total-intensity data.

Table 1. RMS misfits (in nT) for field models to the data, calculated assuming (i) isotropic errors ( $\sigma=3 \mathrm{nT}$ ), and (ii) an additional angular uncertainty in the SIM rotation of $\chi=60^{\prime \prime} . e_{F}$ is for the high latitude total intensity data. $\left(e_{X}, e_{Y}, e_{Z}\right)$ are the misfits in the Earth-centred spherical coordinate system. $\left(e_{B}, e_{\perp}, e_{3}\right)$ are respectively the misfits in the main field direction, the direction perpendicular to both the main field and the star camera axis, and the third orthogonal direction.

\begin{tabular}{cllcccccc}
\hline & & $e_{F}$ & $e_{X}$ & $e_{Y}$ & $e_{Z}$ & $e_{B}$ & $e_{\perp}$ & $e_{3}$ \\
\hline (i) Isotropic & (a) May & 3.11 & 5.82 & 5.60 & 5.19 & 2.83 & 8.49 & 3.47 \\
modelling & (b) December & 3.53 & 5.39 & 5.61 & 4.79 & 2.64 & 8.10 & 3.28 \\
\hline (ii) Anisotropic & (a) May & 2.87 & 5.90 & 5.73 & 5.63 & 2.24 & 9.17 & 3.18 \\
modelling & (b) December & 3.39 & 5.50 & 5.70 & 5.11 & 2.00 & 8.68 & 3.10 \\
\hline
\end{tabular}

has been lost. Were the purpose of this work to produce an accurate model of the geomagnetic field, such gaps would be unacceptable; however, within the framework of this study, it was decided to restrict the data to the one (vector) instrument alone. At high latitudes $\left(>50^{\circ}\right)$ only intensity measurements (F) (calculated from the vector data) were used, to limit possible contamination by field aligned currents, and also to mimic standard field modelling treatments as closely as possible. Outliers were removed during the modelling process, resulting in the elimination of some high latitude intensity data, and, for the May data, 10 vector data with extremely large attitude error (probably arising from periods when the star imager (SIM) was not fully functional). The resulting data distributions are shown in Fig. 3.

The modelling technique used is extremely (possibly overly) simple, since the external fields are not parameterised with a Dst dependence (Langel et al., 1996; Olsen et al., 2000), and secular variation over the period of each data set is ignored. The major complication is that the data must be transformed from spacecraft coordinates. The angular transformation between the SIM and CSC is solved for simultaneously with the calculation of a main field model, using transformation programs developed by Dr. Karlheinz Goedderz (GFZ CHAMP team) (see also Olsen et al., 2000). Fortunately, the determination of these angles turns out to be essentially independent of the treatment of attitude error.

I begin by modelling the vector data errors isotropically, and with vector and scalar data weighted equally ( $\sigma=3 \mathrm{nT})$. The fits of the models to the data are listed in Table 1(i). The results for the two data sets are remarkably similar.

In the standard Earth centred spherical coordinate system, where $(X, Y, Z)$ are North, East, and Down, the misfit to the data is of order 5-6 nT for each component. However, assuming that the predicted attitude error is indeed the major cause of error, it is instructive to examine the misfit to the data in the natural coordinate system. The three axes are given by the vectors forming the dyadics of the covariance matrix in Eq. (9), namely the direction of the field (denoted $B$ ), the direction perpendicular to both the field and the SIM boresight (denoted $\perp$ ), and the third direction making up the orthogonal set (denoted 3 ). This reference frame obviously depends on both the direction of the field and the direction of the SIM, and so is newly defined for each data point. The much larger error in the perpendicular direction is obvious. This error, in combination with the field geometry and star camera direction, also explains the lower value of $e_{Z}$ relative to $e_{X}$ and $e_{Y}$. These results are in agreement with those of Olsen et al. (2000) who first commented on the error anisotropy; note, however, that they use a slightly different coordinate system.

Prompted by these results, I generate a second field model, explicitly considering this error anisotropy (statistics listed in Table 1(ii)). As $e_{3}$ is not much greater than $e_{B}$, I assume for simplicity that pointing error is negligible, and adopt the simplified data covariance matrix (10), assuming $\chi=60^{\prime \prime}$. This value was inspired by the scatter about a smooth curve fit to SIM data from early in the mission. This high value is justified a posteriori from the modelling below, and discussed further in Section 7. As data in the $\perp$ direction is now given a lower weighting, the new model has a higher value of $e_{\perp}$. In other words, as would be expected, modelling the anisotropy slightly increases the anisotropy observed in the residuals. The misfit to the cartesian components is also increased, but the total intensity data and well-determined eigenvector directions are better fit. The fits to the vector 

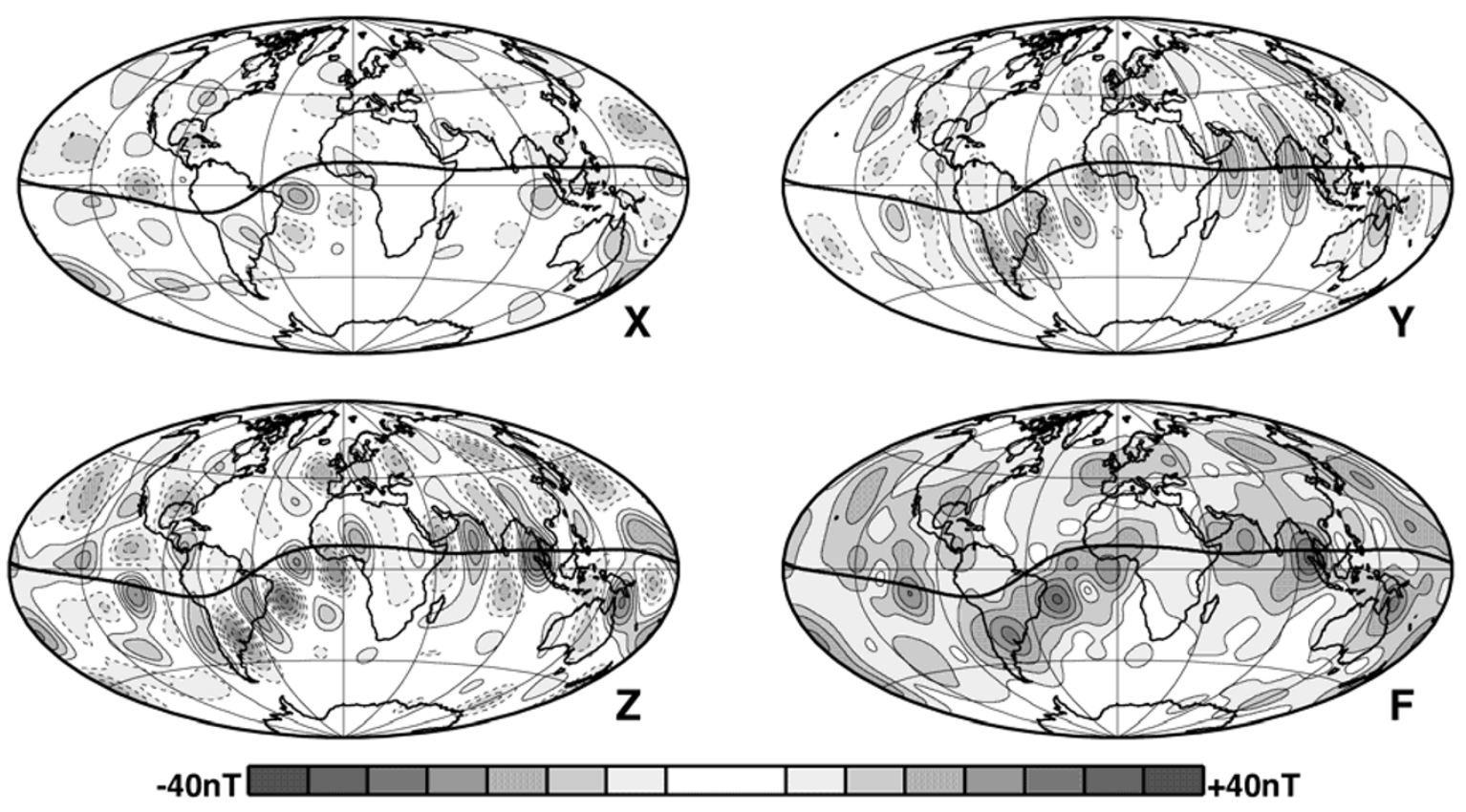

Fig. 4. Plot of differences in the three magnetic components between the two models at the Earth's surface. Dotted contours denote negative values. The solid line marks the dip equator. The $F$ plot is the rms total vector error, not the difference between total intensities.

data field magnitude (2.24 nT in May, and $2.00 \mathrm{nT}$ in December) are particularly good. The rms vector difference between the two models at the Earth's surface is $10.7 \mathrm{nT}$, and it peaks at over $30 \mathrm{nT}$. Differences of this magnitude are very important; for example, they are comparable in magnitude to large crustal anomalies.

In Fig. 4, I plot the difference in the field models for May of Tables 1(ia) and (iia). The $X, Y$, and $Z$ plots are the differences in these components, and $F$ the rms value of these three components (not the difference in the value of the total intensity between the two models). The pattern of the difference arises from several effects. First, there are large differences in areas with data gaps (for example, the South Atlantic anomaly), where the field model is controlled by the fit to data around the edges of the gaps. Second, the largest changes are close to the magnetic equator. As the star camera lies in the plane perpendicular to the orbit, and the field is (by definition) horizontal, the large attitude error direction is approximately vertical. This gives rise to a weak form of the Backus effect or perpendicular error effect (Stern and Bredekamp, 1975; Lowes 1975), which is well known to be most significant near the magnetic equator. Third, other strong differences (in the South Pacific and the South East United States) are in regions where the magnitude of $\hat{\mathbf{n}} \wedge$ $\mathbf{B}$ is particularly large, and so the effects of attitude error particularly significant.

Is the predicted error of $60^{\prime \prime}$ consistent with the data residuals? To address this question I examine the statistics of the data misfit in the three orthogonal directions $(B, \perp, 3)$. If the various errors are assumed of known distribution (most simply, normally distributed), then estimates of $\sigma, \chi, \psi$ can be obtained from the data residuals by the solution of the appropriate maximum likelihood problem; this procedure is described in Appendix C. For greater accuracy, all available data, not just the decimated data, were used (although the
Table 2. Estimates for isotropic and attitude standard deviation from maximum likelihood analysis for full $1.13 \mathrm{~s}$ data.

\begin{tabular}{lcccc}
\hline & \# data & $\sigma$ & $\chi$ & $\psi$ \\
\hline May & 36942 & $2.2 \mathrm{nT}$ & $75^{\prime \prime}$ & $17^{\prime \prime}$ \\
December & 49082 & $1.9 \mathrm{nT}$ & $75^{\prime \prime}$ & $18^{\prime \prime}$ \\
\hline
\end{tabular}

decimated data give very similar results). The results are presented in Table 2. The different $\sigma$ values between May and December probably reflect different levels of magnetic activity (noise from external fields) during the two periods, but the angular parameters remarkably consistent, if slightly larger than expected. This may be due to non-normality of the errors; in particular, it is not clear that the uncertainty in the star camera angles will necessarily be normally distributed, and longer-tailed distributions would increase the variance estimates. In Fig. 5 I plot the distributions of the weighted residuals against a normal curve for comparison. The general shape of the histograms is correct, although the high peaks in the $e_{B}$ and $e_{\perp}$ directions suggest long tails to the distribution, resulting in an increased estimate of the variances.

The argument presented here is slightly inconsistent, as I have (for example) estimated $\psi$ from residuals obtained from modelling assuming its value to be 0 . However, using residuals from modelling assuming isotropic errors produces similar results, so I believe the values presented here to be at least of the right order of magnitude. These values suggest that modelling non-zero $\psi$ (from attitude error or field aligned currents) could be useful, although because the magnitude of the resulting attitude error is not much greater than the isotropic error, the effect on the calculated field model is likely to be small (see Paper 2). 
B direction

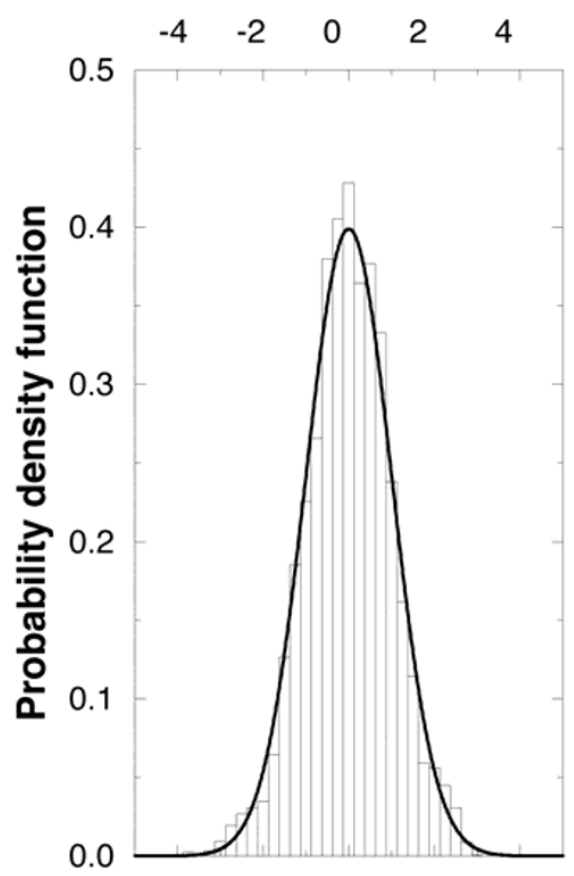

Perp direction

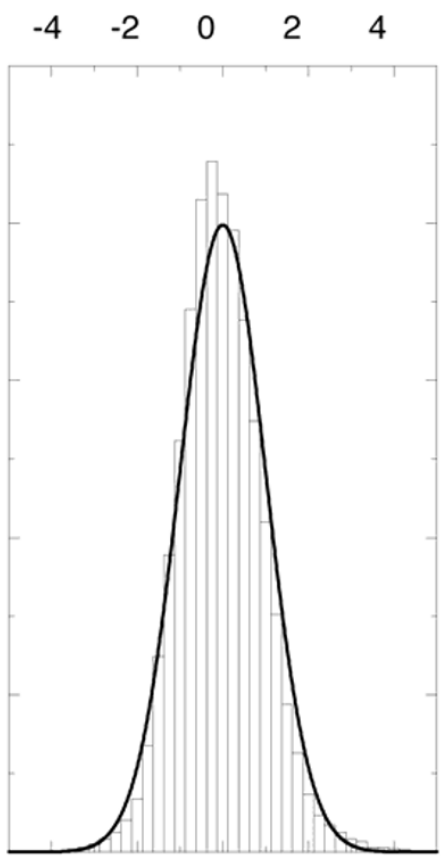

3rd direction

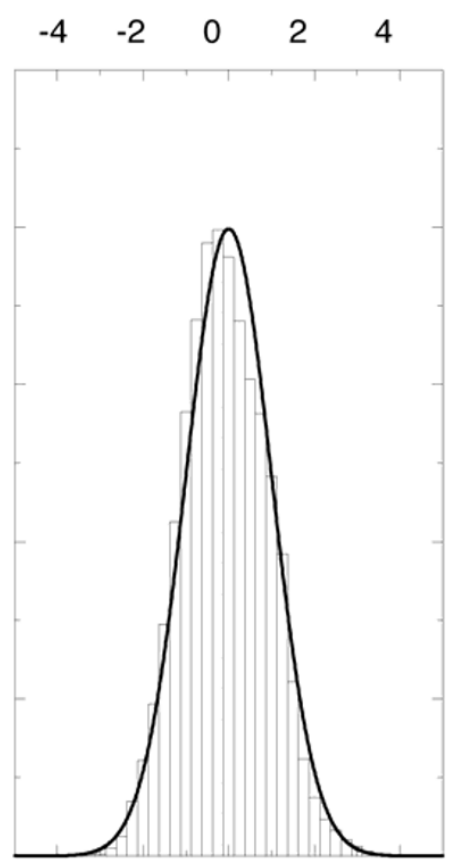

Fig. 5. Histograms of the normalised data misfits for the December data in the three perpendicular directions, with each residual normalised to unit variance with the parameter values given in Table 2 . The continuous curves are predictions assuming a normal distribution.

Table 3. Results for simulations of attitude errors. Values listed are rms differences between the true model, and the estimate derived from modelling noisy synthetic data. Averages of 1000 simulations for the data distribution of the May data set. Misfits are rms misfit to the input model at the Earth's surface (Eq. (12)). The percentage quantity in brackets is the worsening of the solution over the optimal arising from incorrect treatment of attitude error.

\begin{tabular}{ccccc}
\hline \multicolumn{2}{c}{ Input errors } & \multicolumn{3}{c}{ Assumed modelling error } \\
$\sigma$ & $\chi$ & \multicolumn{1}{c}{$0^{\prime \prime}$} & \multicolumn{1}{c}{$30^{\prime \prime}$} & $60^{\prime \prime}$ \\
\hline $2 \mathrm{nT}$ & $60^{\prime \prime}$ & $8.04 \mathrm{nT}(64 \%)$ & $5.25 \mathrm{nT}(7 \%)$ & $4.91 \mathrm{nT}$ \\
$2 \mathrm{nT}$ & $30^{\prime \prime}$ & $5.32 \mathrm{nT}(16 \%)$ & $4.58 \mathrm{nT}$ & $4.74 \mathrm{nT} \mathrm{(3 \% )}$ \\
$2 \mathrm{nT}$ & $0^{\prime \prime}$ & $4.03 \mathrm{nT}$ & $4.33 \mathrm{nT}(7 \%)$ & $4.69 \mathrm{nT}(16 \%)$ \\
$5 \mathrm{nT}$ & $60^{\prime \prime}$ & $12.23 \mathrm{nT}(10 \%)$ & $11.48 \mathrm{nT}(3 \%)$ & $11.16 \mathrm{nT}$ \\
$5 \mathrm{nT}$ & $30^{\prime \prime}$ & $10.67 \mathrm{nT}(1 \%)$ & $10.53 \mathrm{nT}$ & $10.73 \mathrm{nT}(2 \%)$ \\
$5 \mathrm{nT}$ & $0^{\prime \prime}$ & $10.10 \mathrm{nT}$ & $10.20 \mathrm{nT}(1 \%)$ & $10.59 \mathrm{nT}(5 \%)$ \\
\hline
\end{tabular}

The misfit in the $\hat{\mathbf{B}}$ direction $\left(e_{B}\right)$ is surprisingly small, and approaches the expected contribution from the crustal field (degrees 14 and higher) of around $2 \mathrm{nT}$. With better modelling of external and ionospheric fields, it may become important to consider error anisotropy arising from crustal contamination. This is outlined briefly in Appendix D.

\section{Simulations}

In the previous section, I have shown that modelling for attitude error in Ørsted data produces a large (over $30 \mathrm{nT}$ for this example) change in the field model. However, this does not prove that the change is an improvement. To investigate this, I use simulations to examine the penalty resulting from modelling the data incorrectly. I take the identical data distribution (in scalar and vector data) from the real May dataset described above, and using the model determined from those data, calculate synthetic data, as in Section 4 adding both isotropic and attitude Gaussian errors. I consider an isotropic error $\sigma$ of $2 \mathrm{nT}$ or $5 \mathrm{nT}$ (covering the optimistic and pessimistic ends of what could be achievable with Ørsted data) and attitude errors of $\chi=0^{\prime \prime}, 30^{\prime \prime}$, and $60^{\prime \prime}$. For simplicity, $\psi$ is set to 0 . A model is then fit to the data assuming attitude errors of $0^{\prime \prime}, 30^{\prime \prime}$, and $60^{\prime \prime}$. I average the results of 1000 simulations, and present the results in Table 3, giving again the rms misfit to the input field model at the Earth's surface. As usual, the absolute values depend on the simulation details, but relative values should be robust. An incorrect estimate of the attitude error increases model misfit. For ease of comparison I include the percentage increase of the misfit from the correct (self-consistent) modelling.

As might be expected, the results for the extreme case are most striking; with attitude error of $\chi=60^{\prime \prime}$ and isotropic error of $\sigma=2 \mathrm{nT}$, failure to model the attitude error increases the model misfit by $64 \%$. However, from Table 2, such "extreme" values seem to be those appropriate for both May and December Ørsted data sets! 

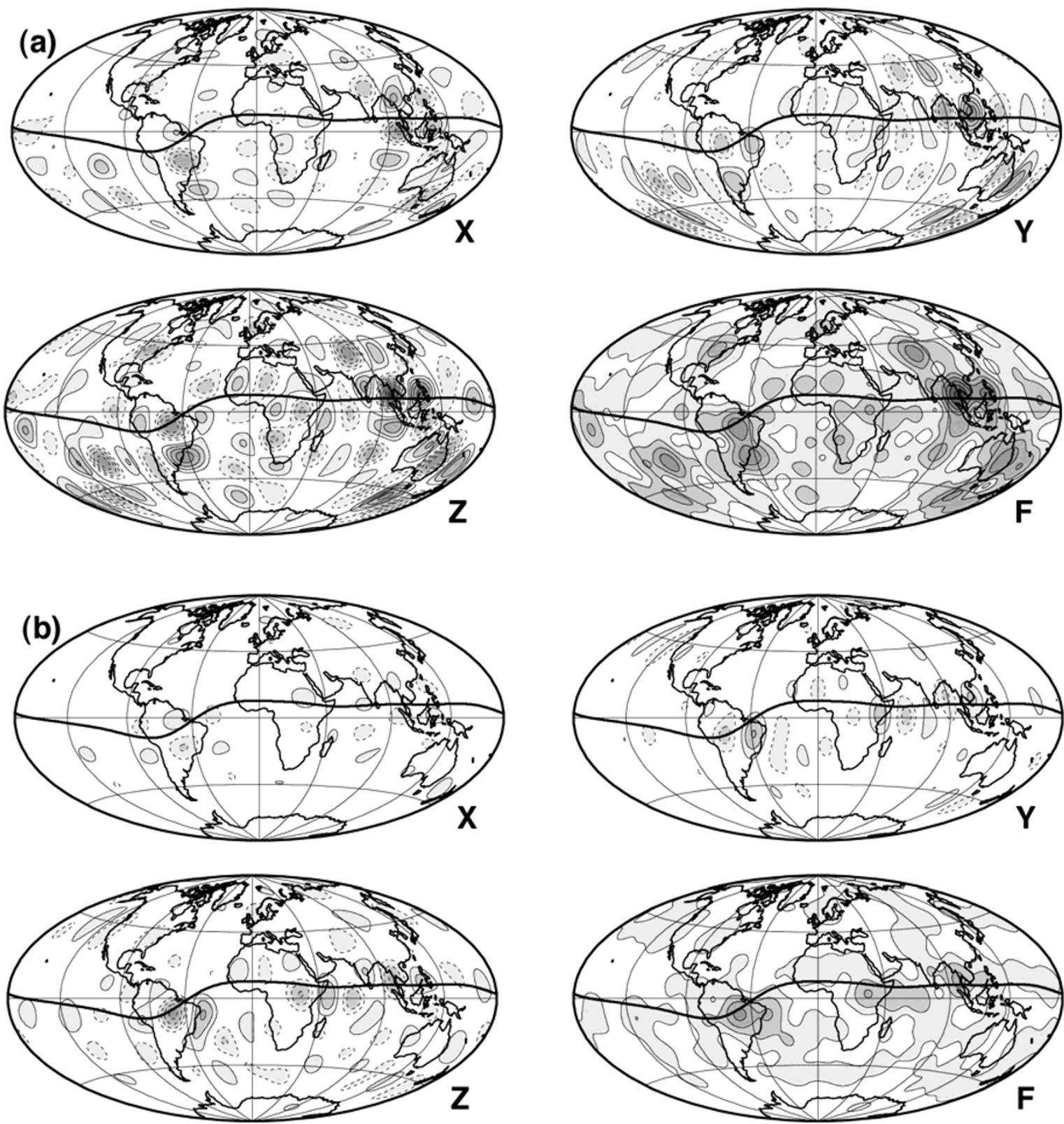

$-40 n T$

Fig. 6. Error distribution for synthetics, input uncertainties $\sigma=2 \mathrm{nT}, \chi=60$ (a) Errors modelled isotropically. (b) Errors modelled correctly. Dashed contours denote negative values.

It is also interesting to examine the spatial distribution of the errors, particularly in comparison with model differences between different error treatments for the real data, plotted in Fig. 4. The error details differ considerably from simulation to simulation, but for an impression of the pattern, I select a characteristic simulation $\left(\sigma=2 \mathrm{nT}, \chi=60^{\prime \prime}\right)$ with errors similar to the mean values. The error distributions for these simulations are plotted in Fig. 6, in (a) with an incorrect (isotropic) error treatment, and in (b) with error covariance correctly modelled. The improvement in model from using a correct error model needs no further emphasis. As with the real data (Fig. 4), the largest model differences are in data gaps, and near the dip equator. However, this error pattern is less clear in the simulation than with the real data. This is probably due to error correlations between locations for the real data (correlated external noise, crustal effects, and especially correlated attitude error).

Table 3 shows that in general, incorrectly estimating the magnitude of the attitude error is less damaging than not modelling it. In particular, little damage should result with the real data from modelling the attitude uncertainty as $\chi=60^{\prime \prime}$ when it should perhaps be $\chi=70^{\prime \prime}$. There is one vector component in which the data are poor; that this component is downweighted is more significant than by how much. In contrast, the penalty (essentially due to an effective reduction in the number of data) from downweighting a direction unnecessarily (when the errors are in reality isotropic), is generally smaller (and much smaller, for example, than with 
isotropic error only using $Z$ data rather than all three components).

\section{Conclusions}

By now, it should be obvious that I recommend modelling of the attitude error in Ørsted data to allow the extraction of the maximum amount of information from the data. Such modelling is particularly important for the data sets considered here, but will also apply even if in future the attitude error can be reduced. If the reader doubts this, consider the alternative. It is customary to regard data with large misfit to the model as outliers, and remove them from the modelling process. However, if the error is not modelled, how is this to be achieved? If attitude error is the cause, and (say) the $Y$ component is compromised, should the $X$ and $Z$ components be trusted? As should hopefully be clear from the formalism, and is discussed in Paper 2, the implementation of the method is straightforward. The only information required in addition to the magnetic field components is the direction of the star camera. Should this for some reason be lacking, I would recommend modelling even Ørsted data as having isotropic attitude error (Eq. (8)). The loss of information from the 3rd direction is more than compensated for by the removal of the biasing $\hat{\mathbf{n}} \wedge \mathbf{B}$ direction, and as noted in Section 5, there is evidence of some attitude error, or attitude-like field-aligned current error, in the 3rd direction. As more data become available, it could prove fruitful to model this error as well; indeed, parameterising for field-aligned currents using this formalism was previously suggested in Paper 2. However, the anisotropy due to this direction is smaller than the rotational direction, and so the effect on the field model is much smaller.

Why should $\chi$ be so large? At time of writing, this has not been explained. Pre-flight tests of the SIM suggested a much smaller value; uncertainties of $2-3^{\prime \prime}$ in the pointing direction, and $10-18^{\prime \prime}$ in rotation, and it appears to be performing according to these specifications (J. L. Jørgensen, pers. comm.). One possibility would be a small error in the timing calibration between the SIM and CSC, although this will not account for the whole effect. The error is certainly not completely random; there is evidence of correlation in the error between successive orbits (Nils Olsen, pers. comm.), and a plot of $e_{\perp}$ shows significant power over a period of about 8 minutes; interestingly, this is the approximate time for a star to cross the field of view of the camera.

I have presented a statistical method to deal with the attitude uncertainty arising from the anisotropy of the Ørsted star camera, so as to produce the minimum contamination in main field modelling. However, ideally it would be best to eliminate or reduce the uncertainty, rather than have to model it. Efforts are continuing to characterise, explain and eliminate this error at source, and attempts are being made to smooth the attitude data, although its anisotropy and correlated nature make this difficult. These issues are beyond the scope of the current paper. However, a third issue is related to this study, so I mention it here. As noted in the theory section, the effect of the attitude error is highly dependent on the angle between the magnetic field and the SIM. If the SIM axis is aligned with the field, the large attitude uncertainty does not affect the measurements. Currently, the satellite is positioned in its orbit such that the star camera points approximately perpendicular to the orbital plane. This has many operational advantages; for example, it limits the chance of the star camera being blinded by the sun when the satellite is in a noon-midnight orbit. As the magnetic field broadly speaking lies in or close to the orbital plane, the effect of the attitude error is maximised. However, it is planned for operational reasons to rotate the satellite at some point. If this was done so that the SIM axis lay in the plane of the orbit, the effects of uncertainty in the attitude error would be significantly reduced (by reducing the average of $|\hat{\mathbf{n}} \wedge \mathbf{B}|$ over the orbit). This might be particularly useful when the satellite orbit is close to the dawn-dusk meridian (probably at any local time between 3 o'clock and 9 o'clock), when the risk of pointing the star camera towards the sun will be reduced

Acknowledgments. The basic formalism reviewed here was developed in collaboration with Jeremy Bloxham and Andy Jackson Frank Lowes has been especially helpful in forcing me to think in more detail about the problem; he also pointed out that maximum likelihood was the appropriate way to estimate the parameter variances. I was supported for part of this work by a NERC postdoctoral fellowship held at the University of Edinburgh.

This work would naturally have been impossible without the Ørsted project. Nils Olsen has been extremely helpful in providing data, and in answering questions. I am grateful for the support of the Ørsted Project Office and the Ørsted Science Data Centre at the Danish Meteorological Institute. The Ørsted Project is funded by the Danish Ministry of Transport, Ministry of Research and Information Technology, and Ministry of Trade and Industry. Additional support was provided by National Aeronautics and Space Administration (NASA), European Space Agency (ESA), Centre Nationale d'Etudes Spatiales (CNES), and Deutsche Agentur für Raumfahrtangelegenheiten (DARA).

\section{Appendix A. Combined Treatment of Vector and Scalar Satellite Magnetic Data}

Magsat carried both a vector and scalar magnetometer (Langel et al., 1982). When operating correctly, the scalar instrument was twice as accurate as the vector instrument Modelling of Magsat data has used either vector or scalar data, but not both at the same time and place. However, Lowes and Martin (1987) suggested modelling the field using both vector and scalar data, combining the advantages of the two data sets (accurate scalar intensity from the scalar instrument, plus directional information from the vector instrument). They argued that because the vector uncertainties were dominated by attitude uncertainty, error correlations between the scalar and vector data could be neglected. However, with correctly modelled attitude error, correlations will be significant. Here, we describe how to combine the two data sets correctly.

Following Magsat rather than Ørsted, the orientation of the spacecraft is assumed equally well known in all directions, so the appropriate covariance matrix for the vector data is given by Eq. (8). It follows that in the reference frame defined by the field, the three "field components" (the field strength and the two null directions ${ }^{1}$ ) are independent. Hence, assuming that the sampling times of the two data sets are the same, the

\footnotetext{
${ }^{1}$ This can be thought of in terms of modelling the data by its magnitude and direction (for example, intensity, declination, and inclination)-compare with equation (A6) in Paper 3.
} 


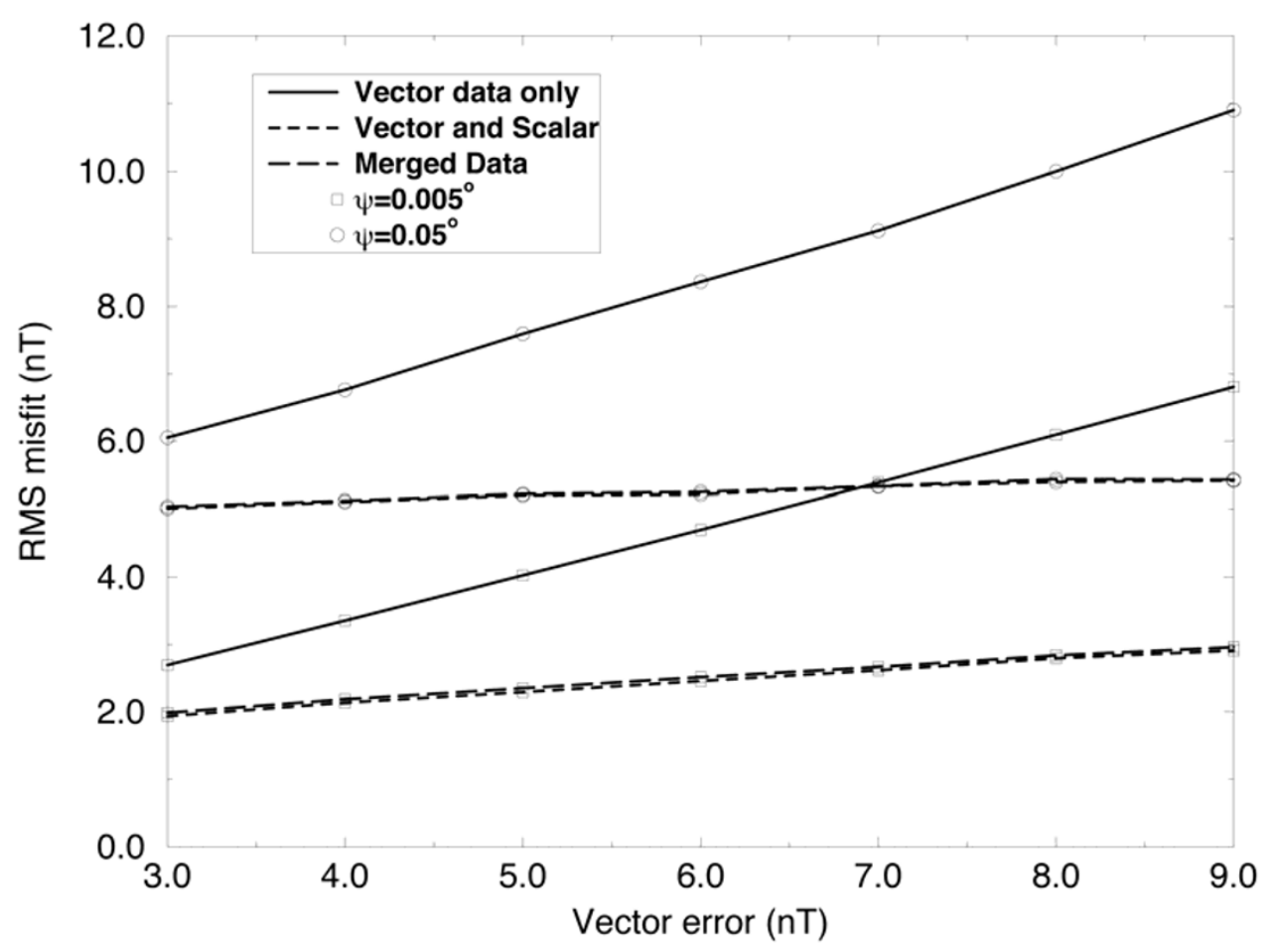

Fig. 7. Simulation of effect of combining vector and scalar data. Each data point is an average of 100 simulations. Scalar data accuracy 2 nT, vector data as given by axis, attitude by symbol type.

measurement of total intensity from the vector instrument can simply be replaced by a best estimate combined from the vector and scalar data. If the two measurements are uncorrelated (errors in the data are dominated by instrumental noise rather than external fields or unmodelled crustal field, for example), this best estimate of the field is one with direction measured by the vector instrument, but with amplitude $F$ calculated from a suitably weighted combination of the vector and scalar measurements:

$$
\mathbf{B}^{\prime}=\frac{F}{|\mathbf{B}|} \mathbf{B} ; \quad F=\frac{\sigma_{s}^{2} \mathbf{B}^{2}+\sigma_{v}^{2} B_{s}^{2}}{\sigma_{s}^{2}+\sigma_{v}^{2}} ; \quad \sigma_{F}^{2}=\frac{\sigma_{s}^{2} \sigma_{v}^{2}}{\sigma_{s}^{2}+\sigma_{v}^{2}}
$$

where $\mathbf{B}^{\prime}$ is the new field vector, $\mathbf{B}$ the vector measurement, $B_{s}$ the scalar measurement, and $\sigma_{s}^{2}$ and $\sigma_{v}^{2}$ are the measurement uncertainties of the scalar and vector measurements respectively. The covariance matrix is then

$$
\begin{aligned}
\mathbf{C}= & \sigma_{F}^{2} \frac{\mathbf{B B}^{\mathrm{T}}}{B^{2}}+\left(\sigma_{v}^{2}+B^{2} \psi^{2}\right) \frac{(\hat{\mathbf{n}} \wedge \mathbf{B})(\hat{\mathbf{n}} \wedge \mathbf{B})^{\mathrm{T}}}{(\hat{\mathbf{n}} \wedge \mathbf{B})^{2}} \\
& +\left(\sigma_{v}^{2}+B^{2} \psi^{2}\right) \frac{(\mathbf{B} \wedge(\hat{\mathbf{n}} \wedge \mathbf{B}))(\mathbf{B} \wedge(\hat{\mathbf{n}} \wedge \mathbf{B}))^{\mathrm{T}}}{(\mathbf{B} \wedge(\hat{\mathbf{n}} \wedge \mathbf{B}))^{2}} .
\end{aligned}
$$

If $\sigma_{s}<\sigma_{v}$ (for Magsat, $\sigma_{v} \approx 2 \sigma_{s}$ ), a significant improvement in model quality could be expected from using this form of the data and covariance matrix. Note also that the scalar (intensity) data are incorporated while keeping the modelling linear. This consideration is not important for main field modelling, where with modern computing the solution for, e.g., $l \max =13$ is very rapid. However, with much higher truncation levels (such as the degree 63 model of Cain et al. (1989)) to include crustal fields, a linear problem with a unique solution which can be determined without iteration is still very valuable.
To confirm that the formalism works, I consider some simulations, similar to those described above in Section 4, but with added scalar data at each point. Data are synthesised with scalar data error $\sigma=2 \mathrm{nT}$, isotropic attitude error of either $\psi=0.005^{\circ}$ or $\psi=0.05^{\circ}$, and vector data errors $\sigma_{v}$ from $3 \mathrm{nT}$ to $9 \mathrm{nT}$. In each case, three simulations are considered, the first with vector data only, the second with vector data and scalar data at each point (considered independent), and third with the data combined as described in this section. The resulting errors are plotted in Fig. 7. As earlier, the absolute values of the misfits are not meaningful, only the ratio of misfits between the different curves. In both cases the addition of scalar data significantly improves the model, and there is little difference between considering the scalar data separately or with the new formalism (the small difference probably arises from the fact that the errors in the new formalism are no longer exactly Gaussian). It might seem that scalar and vector data can be considered together, as advocated by Lowes and Martin (1987), but this ideal case includes no correlations between the two instruments. In practice, noise sources (for example, external fields) are likely to be correlated, and this can be treated much more simply in the new formalism (essentially by just increasing the variance estimate) than by formally considering the correlation between separate scalar and vector data.

This formalism allows for the inclusion of the highly accurate scalar intensity data, as well as directional information, without redundancy in measurements. For Magsat, we might expect some correlation between vector and scalar measurements, as the scalar magnetometer was used to correct the drift in the vector fluxgate magnetometer, amounting to $20 \mathrm{nT}$ over the lifetime of the satellite (Langel et al., 1982). How- 
ever, that drift was slow, and is calculated from many vector and scalar data. Thus, the resulting correlation between individual data from the two data sets should be negligible. However, if correlation is a worry, or the noise in the vector measurements is much larger than that in the scalar measurements, it may be better just to adopt the scalar measurement $B_{s}$ for $F$ along with its associated standard deviation $\sigma_{s}$.

This problem is not purely of historical interest. The Cassini mission to Saturn will provide similar data: at closest approach, the proton magnetometer is to be switched to scalar mode, but less accurate vector measurements will be provided by the fluxgate magnetometer (Dunlop et al., 1999). The production of the best possible models of the Saturnian field will require correct treatment of these data.

\section{Appendix B. Using Only Directional Information from Three Component Data}

It is well known that field modelling using total intensity data only is compromised by the perpendicular error or Backus effect (Stern and Bredekamp, 1975; Lowes, 1975). When vector data are not available (for example, due to a satellite carrying only a scalar magnetometer, or because of gaps in attitude information as found for Ørsted over the South Atlantic anomaly), it is common to try to replace these data, for example either by using data from other epochs forward or backward continued with a model of secular variation, or by construction of synthetic data (see, for example, Macmillan and Quinn, 2000). However, such "data" are obviously less reliable than real data. Only the vector information is required; the scalar intensity of the data should not be considered as an alternative to real measured scalar intensity. This could be accomplished by using declination and inclination values. A simple alternative (which also keeps the modelling problem linear) is to downweight the field magnitude information of the synthetic data. For simplicity assuming isotropic attitude error (Eq. (7)), this can be achieved with the covariance matrix

$$
\mathbf{C}=\sigma^{2} \mathbf{I}+\psi^{2}\left(B^{2} \mathbf{I}-\mathbf{B B}^{\mathrm{T}}\right)+\left(\frac{\omega^{2}}{B^{2}}\right) \mathbf{B B}^{\mathrm{T}}
$$

where $\omega \gg \sigma, B \psi$ is a very large number which ensures that the modelled variance of the synthetic data in the direction of the main field is enormous, and therefore that this information does not influence the model. In my preferred dyadic form, this matrix is written

$$
\begin{aligned}
\mathbf{C}= & \left(\sigma^{2}+\omega^{2}\right) \frac{\mathbf{B B}^{\mathrm{T}}}{B^{2}}+\left(\sigma^{2}+B^{2} \psi^{2}\right) \frac{(\hat{\mathbf{n}} \wedge \mathbf{B})(\hat{\mathbf{n}} \wedge \mathbf{B})^{\mathrm{T}}}{(\hat{\mathbf{n}} \wedge \mathbf{B})^{2}} \\
& +\left(\sigma^{2}+B^{2} \psi^{2}\right) \frac{(\mathbf{B} \wedge(\hat{\mathbf{n}} \wedge \mathbf{B}))(\mathbf{B} \wedge(\hat{\mathbf{n}} \wedge \mathbf{B}))^{\mathrm{T}}}{(\mathbf{B} \wedge(\hat{\mathbf{n}} \wedge \mathbf{B}))^{2}}
\end{aligned}
$$

(compare with Eq. (8)).

\section{Appendix C. Maximum Likelihood Estimation of Variances}

We wish to estimate from the data residuals the three parameters $\sigma, \psi, \chi$ as defined in the covariance matrix (9). The errors are supposed uncorrelated and normally distributed, so that

$$
e_{B}=\sigma \mathcal{N}(0,1)
$$

$$
\begin{aligned}
e_{\perp} & =\sigma \mathcal{N}(0,1)+\chi|\hat{\mathbf{n}} \wedge \mathbf{B}| \mathcal{N}(0,1)+\psi(\hat{\mathbf{n}} . \mathbf{B}) \mathcal{N}(0,1) \\
e_{3} & =\sigma \mathcal{N}(0,1)+\psi B \mathcal{N}(0,1) .
\end{aligned}
$$

To simplify notation, define

$$
B_{\perp}=|\hat{\mathbf{n}} \wedge \mathbf{B}| ; \quad B_{\|}=\hat{\mathbf{n}} . \mathbf{B} .
$$

First, consider only the $e_{\perp} \equiv X$ direction. The probability that a particular misfit $X_{i}$ lies in the range $X$ to $X+\Delta X$ is given by the appropriate normal probability density function

$$
\frac{\exp \left[-X^{2} /\left(2\left(\sigma^{2}+\chi^{2} B_{\perp}^{2}+\psi^{2} B_{\|}^{2}\right)\right)\right]}{\sqrt{2 \pi\left(\sigma^{2}+\chi^{2} B_{\perp}^{2}+\psi^{2} B_{\|}^{2}\right)}} d X .
$$

The likelihood function that a particular set of $n$ observations $\left\{X_{i}\right\}$ arises from such a distribution with defined $\sigma, \psi, \chi$ is then given by

$$
\begin{aligned}
& L(\sigma, \psi, \chi) \\
& \quad=\prod_{i=1}^{n} \frac{\exp \left[-X_{i}^{2} /\left(2\left(\sigma^{2}+\chi^{2} B_{\perp_{i}}^{2}+\psi^{2} B_{\|_{i}}^{2}\right)\right)\right]}{\sqrt{2 \pi\left(\sigma^{2}+\chi^{2} B_{\perp_{i}}^{2}+\psi^{2} B_{\|_{i}}^{2}\right)}} d X .
\end{aligned}
$$

Given a particular data set, the maximum likelihood estimates of $\sigma, \psi, \chi$ are those which maximise this function, or equivalently minimise the function

$$
\begin{aligned}
& -\ln [L(\sigma, \psi, \chi)] \\
& =\frac{1}{2} \sum_{i=1}^{n}\left[\ln \left(\sigma^{2}+\chi^{2} B_{\perp_{i}}^{2}+\psi^{2} B_{\|_{i}}^{2}\right)\right. \\
& \left.\quad+\frac{X_{i}^{2}}{\sigma^{2}+\chi^{2} B_{\perp_{i}}^{2}+\psi^{2} B_{\|_{i}}^{2}}\right]+ \text { constants. }
\end{aligned}
$$

Seeking a local extremum of the function (C.5) by standard partial differentiation methods yields the following three equations:

$$
\begin{aligned}
& \frac{\partial}{\partial \sigma}: \sum_{i=1}^{n}\left(\frac{1}{\sigma^{2}+\chi^{2} B_{\perp_{i}}^{2}+\psi^{2} B_{\|_{i}}^{2}}\right. \\
& \left.-\frac{X_{i}^{2}}{\left(\sigma^{2}+\chi^{2} B_{\perp_{i}}^{2}+\psi^{2} B_{\|_{i}}^{2}\right)^{2}}\right)=0 \\
& \frac{\partial}{\partial \chi}: \sum_{i=1}^{n}\left(\frac{B_{\perp_{i}}^{2}}{\sigma^{2}+\chi^{2} B_{\perp_{i}}^{2}+\psi^{2} B_{\|_{i}}^{2}}\right. \\
& \left.-\frac{X_{i}^{2} B_{\perp_{i}}^{2}}{\left(\sigma^{2}+\chi^{2} B_{\perp_{i}}^{2}+\psi^{2} B_{\|_{i}}^{2}\right)^{2}}\right)=0 \\
& \frac{\partial}{\partial \psi}: \sum_{i=1}^{n}\left(\frac{B_{\|_{i}}^{2}}{\sigma^{2}+\chi^{2} B_{\perp_{i}}^{2}+\psi^{2} B_{\|_{i}}^{2}}\right. \\
& \left.-\frac{X_{i}^{2} B_{\|_{i}}^{2}}{\left(\sigma^{2}+\chi^{2} B_{\perp_{i}}^{2}+\psi^{2} B_{\|_{i}}^{2}\right)^{2}}\right)=0 .
\end{aligned}
$$

Then, taking $\sigma^{2} \times(\mathrm{C} .6)+\chi^{2} \times(\mathrm{C} .7)+\psi^{2} \times(\mathrm{C} .8)$ gives

$$
\frac{1}{n} \sum_{i=1}^{n} \frac{X_{i}^{2}}{\sigma^{2}+\chi^{2} B_{\perp_{i}}^{2}+\psi^{2} B_{\|_{i}}^{2}}=1
$$


showing that the maximum likelihood estimate gives the mean value of the weighted variance as unity, as required. This expression can also easily be seen to be consistent with various limiting cases; for example, if $\chi=\psi=0$, then

$$
\sigma^{2}=\sum_{i=1}^{n} X_{i}^{2} / n
$$

the standard expression for the sample variance.

Solution of the equation system ((C.6)-(C.8)) is not possible in closed form; in practice it is easiest to seek estimates of $\sigma, \psi, \chi$ by direct numerical minimisation of Eq. (C.5). Because the "data" are inherently noisy (by definition, the signal to noise ratio of one datum is unity) all three error directions are used to obtain the parameters. Thus, writing $Y \equiv e_{3}$ and $Z \equiv e_{B}$, the generalisation of Eq. (C.5) which is minimised in this paper to estimate the parameters is

$$
\begin{aligned}
& f(\sigma, \chi, \psi)=\sum_{i=1}^{n}\left[\ln \left(\sigma^{2}+\chi^{2} B_{\perp_{i}}^{2}+\psi^{2} B_{\|_{i}}^{2}\right)\right.\left.+\frac{X_{i}^{2}}{\sigma^{2}+\chi^{2} B_{\perp_{i}}^{2}+\psi^{2} B_{\|_{i}}^{2}}\right]+ \\
& \sum_{i=1}^{n}\left[\ln \left(\sigma^{2}+\psi^{2} B_{i}^{2}\right)+\frac{Y_{i}^{2}}{\sigma^{2}+\psi^{2} B_{i}^{2}}\right] \\
&+\sum_{i=1}^{n}\left[\ln \left(\sigma^{2}\right)+\frac{Z_{i}^{2}}{\sigma^{2}}\right] .
\end{aligned}
$$

\section{Appendix D. Crustal Complications}

As discussed in Paper 3 and a number of other references, unmodelled crustal field creates an anisotropic contribution to the field error; the error in the vertical direction is $\sqrt{2} \times$ that in either horizontal direction. For high-degree models, when the crustal field is included, this effect can be ignored, as external fields will provide the largest source of error in the measurement. For lower-degree truncated models, however, even at Ørsted altitude, crustal error may be important. Then, for no attitude error, the covariance matrix would be

$$
\hat{\mathbf{x}} \hat{\mathbf{x}}^{\mathrm{T}} \sigma^{2}+\hat{\mathbf{y}} \hat{\mathbf{y}}^{\mathrm{T}} \sigma^{2}+\hat{\mathbf{z}} \hat{\mathbf{z}}^{\mathrm{T}} 2 \sigma^{2}
$$

where $(\hat{\mathbf{x}}, \hat{\mathbf{y}}, \hat{\mathbf{z}})$ are the unit vectors in the North, East, and Down directions. In Paper 3, it was demonstrated that a 5\% degradation of field model quality would be likely if this error was not properly accounted for. In combination with attitude error for Ørsted, the correct covariance matrix becomes

$$
\mathbf{C}=\sigma^{2} \frac{\mathbf{B B}^{\mathrm{T}}}{B^{2}}+\left(\sigma^{2}+(\hat{\mathbf{n}} \wedge \mathbf{B})^{2} \chi^{2}+(\hat{\mathbf{n}} \cdot \mathbf{B})^{2} \psi^{2}\right)
$$

$$
\begin{aligned}
& \cdot \frac{(\hat{\mathbf{n}} \wedge \mathbf{B})(\hat{\mathbf{n}} \wedge \mathbf{B})^{\mathrm{T}}}{(\hat{\mathbf{n}} \wedge \mathbf{B})^{2}} \\
& +\left(\sigma^{2}+B^{2} \psi^{2}\right) \frac{(\mathbf{B} \wedge(\hat{\mathbf{n}} \wedge \mathbf{B}))(\mathbf{B} \wedge(\hat{\mathbf{n}} \wedge \mathbf{B}))^{\mathrm{T}}}{(\mathbf{B} \wedge(\hat{\mathbf{n}} \wedge \mathbf{B}))^{2}} \\
& +\sigma^{2} \hat{\mathbf{z}} \hat{\mathbf{z}}^{\mathrm{T}} .
\end{aligned}
$$

It is possible to calculate an analytic inverse for this matrix, but it is very messy, and a numerical calculation is almost certainly more useful.

\section{References}

Cain, J. C., Z. Wang, C. Kluth, and D. R. Schmitz, Derivation of a geomagnetic model to $n=63$, Geophys. J., 97, 431-441, 1989.

Dunlop, M. W., M. K. Dougherty, S. Kellock, and D. J. Southwood, Operation of the dual magnetometer on Cassini: science performance, Planet. Space Sci., 47, 1389-1405, 1999.

Gubbins, D. and J. Bloxham, Geomagnetic field analysis-III. Magnetic fields on the core-mantle boundary, Geophys. J. R. Astron. Soc., 80, 695$713,1985$.

Holme, R. and J. Bloxham, Alleviation of the Backus effect in geomagnetic field modelling, Geophys. Res. Lett., 22, 1641-1644, 1995.

Holme, R. and J. Bloxham, The treatment of attitude errors in satellite geomagnetic data, Phys. Earth Planet. Int., 98, 221-233, 1996.

Holme, R. and A. Jackson, The cause and treatment of anisotropic errors in near-Earth geomagnetic data, Phys. Earth Planet. Int., 103, 375-388, 1997.

Langel, R. A. and R. H. Estes, The near-Earth magnetic field at 1980 determined from Magsat data, J. Geophys. Res., 90, 2495-2509, 1985.

Langel, R. A., G. Ousley, J. Berbert, J. Murphy, and M. Settle, The Magsat mission, Geophys. Res. Lett., 9, 243-245, 1982.

Langel, R. A., T. J. Sabaka, R. T. Baldwin, and J. A. Conrad, The near-Earth magnetic field from magnetospheric and quiet-day ionospheric sources and how it is modeled, Phys. Earth Planet. Int., 98, 235-267, 1996.

Lowes, F. J., Mean-square values on sphere of spherical harmonic vector fields, J. Geophys. Res., 71, 2179, 1966.

Lowes, F. J., Vector errors in spherical harmonic analysis of scalar data, Geophys. J. R. Astron. Soc., 42, 637-651, 1975.

Lowes, F. J. and J. E. Martin, Optimum use of satellite intensity and vector data in modelling the main geomagnetic field, Phys. Earth Planet. Int., 48, 183-192, 1987.

Macmillan, S. and J. M. Quinn, The 2000 revision of the joint UK/US geomagnetic field models and an IGRF 2000 candidate model, Earth Planets Space, 52, this issue, 1149-1162, 2000.

Olsen, N., T. J. Sabaka, and L. Tøffner-Clausen, Determination of the IGRF 2000 model, Earth Planets Space, 52, this issue, 1175-1182, 2000.

Rygaard-Hjalsted, C., C. G. Constable, and R. L. Parker, The influence of correlated crustal signals in modelling the main geomagnetic field, Geophys. J. Int., 130, 717-726, 1997.

Shure, L., R. L. Parker, and G. E. Backus, Harmonic splines for geomagnetic modelling, Phys. Earth Planet. Int., 28, 215-229, 1982.

Stern, D. P. and J. H. Bredekamp, Error enhancement in geomagnetic models derived from scalar data, J. Geophys. Res., 80, 1776-1782, 1975.

Whaler, K. A. and D. Gubbins, Spherical harmonic analysis of the geomagnetic field: an example of a linear inverse problem, Geophys. J. R. Astron. Soc., 65, 645-693, 1981.

R. Holme (e-mail: holme@gfz-potsdam.de) 